\title{
Impact of EBP project
}

\author{
Edna Aurelus* \\ College of Nursing and Health Innovation at Arizona State University, USA
}

\begin{abstract}
Problem: Overweight and obesity are significant problems in patients being treated with antipsychotic medications. Obesity has reached epidemic proportions in the United States, affecting over 72 million adults [1]. Obesity related conditions or metabolic syndrome include cardiovascular diseases, diabetes, and certain types of
\end{abstract} cancer are some of the leading cause of preventable death [2].

Design: quasi-experimental.

Purpose: The purpose of this project was to implement an evidence-based educational program in a behavioral health setting with an overall goal of decreasing weight gain in patients who are prescribed antipsychotic medicines.

Methods: A 12 week-long program was designed for people who are prescribed a regimen of antipsychotic medicines. The program included guidelines for regular exercise and diet, along with a weekly 1 -hour session of dietary and exercise education. There were 7 participants at the beginning of the program; 5 completed the program. A pre- and post-program questionnaire was administered to assess attitudes and behaviors about eating.

Findings: Participants experienced statistically significant weight reduction $(\mathrm{P}=.043)$. There were no statistical differences on the participants' waist circumference and BMI. Inconsistent attendance may have negatively impacted the results. However, the results were effective in showing no weight gain among all participants.

Conclusion: An educational program to help prevent weight gain induced by antipsychotic medication can be impactful to the health care system.

\section{Dietary and exercise educational program for patients taking antipsychotic medication}

Obesity has reached epidemic proportions in the United States, affecting over 72 million adults [1]. More than one-third or $35.7 \%$ of adults in the United States are obese [2]. Obesity related conditions include cardiovascular diseases, diabetes, and certain types of cancer are some of the leading cause of preventable death [2]. Although different constructive approaches such as media awareness and local community health education are stressing the importance of healthy diet, obesity remains the gateway to many deadly diseases. Despite these efforts, there was a dramatic increase in obesity in the United States from 1990 through 2010 [2].

Metabolic syndrome has reached $20-25 \%$ of the global population [3]. While individuals in the general population are at risk of developing obesity related conditions or metabolic syndrome, people with mental illness are at even higher risk [3]. Many patients are unaware of the negative metabolic effects while taking antipsychotic medication. The potential of these drugs in reducing patients' metabolism remains a serious problem.

\section{Background and significance}

Obesity is a significant cause of morbidity and mortality. Recent data indicate that Americans are gaining weight at an alarming rate. In fact, data from the Center for Disease Control [2] indicates that the United States (U.S.) obesity rate was $12 \%$ in 1990 and had grown to $23 \%$ by 2005 [4]. The prevalence of obesity among adults continues to be greater than one-third of the population. Obesity has been directly linked to hypertension, diabetes, cancer, and depression [5]. This problem is a threat to health and longevity. There are multiple factors such as health status, source of income, race, and level of education that contribute to the phenomenon of obesity. As a practicing provider, it is impossible to ignore the modifiable epidemic. Drastic measures are warranted by providers to educate patients in reducing comorbidities evoked by obesity. Outcome measurement of education on maintaining ideal weight by increasing physical activities, providing tips on calorie balance, healthy eating, and preventing weight gain will be assessed. According to Park et al. [3] there are a group of metabolic abnormalities, which include weight gain, associated with an increased risk of liver disease, cardiovascular disease, diabetes and stroke that are related with the treatment of antipsychotic medications. The induction of obesity by antipsychotic agents has been documented since the introduction of chlorpromazine in the mid-1950s. According to a recent study by $\mathrm{Wu}$ et al. [6] 8.8\% of patients receiving antipsychotic agents increased their baseline weight by more than $7 \%$. Prescribers need to be knowledgeable about antipsychotics so they can appropriately implement treatment plans for their patients. Atypical antipsychotics have been known to have greater potential for inducing weight gain than conventional antipsychotic medications and vary in their propensity to induce weight gain. However, many patients are unaware of the metabolic side effects that the drugs perpetuate. It is conservatively estimated that people with serious mental illness (SMI) die at least 10-15 years earlier than the general population. This disturbing trend can be prevented.

Correspondence to: Edna Aurelus, College of Nursing and Health Innovation at Arizona State University, USA, Tel 480-427-1430; E-mail: edna.aurelus@gmail.com

Key words: obesity, antipsychotic medication, educational program, metabolic syndrome, BMI

Received: June 14, 2016; Accepted: July 04, 2016; Published: July 07, 2016 
The biggest killer in this population is cardiovascular disease (CVD), given the fact that these premature deaths are not related to suicide [7]. Despite the high prevalence of obesity and its associated harmful health effects, physician frequently fail to counsel patients about nutrition and weight management and frequently report and demonstrate a lack of training and competence in obesity management [8].

\section{Internal evidence}

The medical abnormalities that are associated with obesity can be challenging for patients and their providers. Providers very often lack the time to educate patients due to the high patient acuity. In the clinical setting it was observed that patients were given information regarding their poor food choices. Their lack of knowledge on the importance of physical exercise, healthy diet, and the side effects of their medication were alarming. Therefore, as a primary care practitioner practicing in the psychiatric setting, it is almost impossible to ignore this modifiable problem. The metabolic effect of antipsychotic medications led to the question: In adult patients who are taking antipsychotic medication with a serious side effect of weight gain, how does an educational program on healthy diet and exercise regimen compared to standard care be effective in preventing the increasing rates of overweight and obesity?

\section{Synthesis of evidence}

Multiple studies were reviewed, but 10 were selected for this project, as seen on the synthesis table on appendix A. Most of the studies reveal a high level of evidence and revealed positive effectiveness of educational intervention on patients receiving antipsychotic medications, with the exception of one study that suggests positive result from behavioral intervention [9]. All of the studies included negative effects of atypical antipsychotic medications on patients' body mass index. Several of the studies reveal weight improvement with an intervention lasting more than 12 weeks. However, several of the studies that were reviewed and were no longer than 12 weeks reveal little to no weight reduction due to the short duration of the studies. Unfortunately, the weakness that was found in those studies were that most studies were conducted for a short period of time that is 3-6 months and full results of patients' progress was not monitored. Nevertheless, the results of this study were positively significant that patients' weight were improved with educational intervention.

\section{Purpose}

The purpose of this project is to evaluate an evidence-based educational program in a behavioral health setting with an overall goal of decreasing weight gain in patients who are prescribed antipsychotic medicines.

\section{Conceptual framework}

The Transtheoretical Model created by Prochaska, et al. [10] DiClemente, et al. [11] was adapted due to its reliability and effectiveness in improving physicians' practice. The conceptual framework for guidelines using the Transtheoretical Model includes five sample steps and once followed, practitioners would be able to see effectiveness in the evidence base practice adoption. The following are the five steps: Step 1 assessment of practitioner's stage of readiness to change, Step 2: assessment of specific barriers to guideline use, Step 3: determination of appropriate level of intervention, Step 4: design of dissemination and implementation strategies, step 5: evaluation of the implementation strategies [12]. A central tenet of health education theory, and a concept, which is incorporated into the Transtheoretical Model, one cannot change behavior without putting in place attention to gaps in both knowledge and skills [12]. Providers are in the forefront of executing these changes in patients' behaviors. Therefore, it is imperative that providers assess patients' knowledge level about their prescribed medications in order to avoid negative preventable side effects.

\section{Evidence-based practice model}

Mental health nurses need to develop new competencies because they are in a unique position to positively affect the physical health of people who are severely mentally ill (SMI) [13]. The Iowa Model of Evidence-Based Practice (EBP), seen in the figure on appendix D, was selected due to its ability to allow nurses to make decisions about daily practices that affect patient care outcomes. The Iowa Model identifies problems experienced in the clinical setting, and then allows nurses to review, research, intervene and implement a solution to the problem. This model contains five steps that identify promotion of quality care to patients. The following are the different steps. Step one: triggers, which are comprised of problem base or knowledge base, priority of organization team and team formation. Step 2: evidence, which includes searching, evaluating and synthesizing the available articles. Step 3: practice changes, which contain protocol, outcome, and baseline data, implement, evaluate and process. Step 4: adopt practice change and step 5: disseminate [5]. The EBP model is well fitted for the project plan because, nurses are in the forefront among the interdisciplinary team and are able to provide one on one health education. Furthermore, the problem regarding obesity requires immediate health intervention in the delivery of quality patient care.

\section{Methods}

A pre and post-program eating questionnaire about participants' eating habits and choices was conducted. Inclusion criteria for the participants are that they are to be 18 years or older, BMI of 25 or higher taking antipsychotic medication, mentally coherent, and gave consent to participate. Participants will include clients who are both inpatients as well as outpatients. The project will consist of a 12-week long educational program designed for people who are prescribed antipsychotic medications. Participants in the program will be provided guidelines for diet and regular exercise. They will meet individually with the program director weekly for 1-hour educational sessions on diet and exercise.

\section{Team}

The members of the team will be comprised of the project coordinator, who is responsible for providing health education on different approaches that participants can take to avoid weight gain, while on antipsychotics. The charge nurses and the security guard will provide access to the participants in the designated area of the program within the facility. The psychiatrists will provide clearance for inpatient participants who are willing to participate in the hourly group session. The registered nurses (RNs) are responsible for assessing participants' readiness to participate in the program. Behavioral health technicians (BHT) will be in charge of escorting participants to and from the group session. Finally, the dietician will be responsible for educating the participants on healthy food choices.

Project proposal was presented to the administrators of the facility, explaining the benefit of a structured educational program on diet and exercise to the psychiatric population. Selecting a champion 
among the administrators was an approach adopted to hasten the process of approval in the facility. The group dynamic of this team is collaborative and task oriented. Working together by utilizing each other's knowledge and expertise will help to accomplish the project goal, which is increasing patient education and health literacy.

\section{Principle}

The institute of medicine (IOM) defines health literacy as the degree to which individuals can obtain, process, and understand the basic health information and services they need to make appropriate health decisions [14]. Health literacy is an important communication system because it has an impact on health outcomes, quality of care, and healthcare costs [15]. One of the principles utilized to plan and implement the project is health literacy. Health literacy is an important part of this project because there is a sense of community among patients and the healthcare team. The team brings together the four E's which are engage, educate, empower, and enable.

\section{Engage}

Participants' involvement in the project is vital for success for weight maintenance. Part of the education program is getting the participants to provide a weekly food journal and discuss their healthy food choices for the week. This will give them a sense of engagement during the process.

\section{Education}

Education is provided on the amount of caloric intake needed for different body types. Exercise regimen will be discussed and demonstrated individually to ensure that participants will correctly perform the exercises. Locations of affordable farmers' markets within the region will be provided for organic food purchase.

\section{Empower and enable}

Participants then use the knowledge tools learned from the program education to feel empowered and abled to make the changes necessary for their health. As a leader promoting healthy lifestyle and disease prevention, developing a dietary and exercise educational program is the approach that will be applied to ensure that participants of the mentioned population are receiving the proper health education.

\section{Outcomes measurement}

A survey including questions to assess participants' demographic characteristic of race, gender, and age will be given to each participant. A food questionnaire, focusing on cognitive dietary restraint, dietary log, and monthly weight measurements will be provided to each participant on the first day of the program. Participants are provided with the three-factor eating questionnaire (pre and post-program), and a revised nine-item validated instrument to assess eating behaviors and weight. Participants' monthly weight will be assessed to monitor their improvement. Demographic information, race, gender and age will be collected. Participants will complete a daily dietary log (pre and post-program). Project evaluation of progress will be assessed by the completion of the program (post-test only). The three major outcomes that will be measured during the three months period of the project will include: 1: Weight at baseline, monthly and the last final day of the program. Each participant's weight will be monitored at the beginning of the program and monthly thereafter. During each weight assessment, participant's BMI will be monitored. The ability to maintain the weight assessed at baseline or lose weight will dictate participants' progress during the program.
2: Monthly survey to evaluate participants' behaviors. This approach is adopted in order to assess participants' ongoing progress in order to explore various points in need of modification. Once report of each survey is examined, evaluation of individual's education growth regarding their diet and exercise regimen will be reassessed by measuring their weight, BMI, and repeating the three-factor food questionnaire to see if there were any changes compared to the initial test. The monthly survey will part of a routine assessment. Patients' goal BMI and frequency of the exercise regimen will be addressed. Participants will have the opportunity to share their progress with the group.

3: Final survey to measure satisfaction with the program and intention to continue the tools learned to sustain the healthy behavior. The tools that will be used to monitor participants' progress will include a dietary and daily exercise record from self-journal. The food frequency questionnaires will be reassessed to monitor if any changes have taken place in relation to healthy eating. Participants' final weight will be monitored and discussed individually to assess if the goals made prior to joining the program have been reached.

The logic model explained in the table on Appendix B is a simple model that exhibits a translucent purpose statement to follow for the educational intervention planned for patients taking antipsychotic medication. The logic model simply offers understanding to how and why patients change their behavior. It also gives a clear understanding depicting the expected outcomes. Inputs are clearly identified and précised. Outputs, which include activities and participants, exhibit the appropriateness of the selected theoretical framework.

\section{Barriers and/or facilitators}

Arizona State University Institutional Review Board's approval was difficult to obtain initially due to additional information requested that caused delay in the anticipated date of implementation of the project. The forms requested from the IRB office were completed promptly with the help of the principle investigator (PI) in order to obtain the approval as soon as possible. Approval was rendered in September 26th, 2013. Initially participant recruitment was difficult because participants were skeptical about allowing others to know about their antipsychotic medications. However, once the program coordinator reassured them that everyone's data would be kept confidential to avoid breaching of privacy, they felt more comfortable to participate. Seven participants agreed to join the program. Flyers were posted around the units, staff members approached clients and phone calls were made to potential participants and their social network in order to recruit for the study.

Banner Behavioral Health approved implementation of the project in their facility while the approval from ASU IRB was still pending. Therefore, there was a delay in collecting data from the interested participants, causing as much as three participants to lose interest. Since implementation of the project, the participants have shown great enthusiasm about participating. The participants completed all data, such as consent, food journal and pre- food frequency questionnaires form. Having all the consented seven participants present every session continues to be a challenge, however at least four participants show up every session.

\section{$\underline{\text { Results }}$}

The twelve weeks study was conducted at Banner Behavioral Health. Seven participants agreed to attend. Two participants declined to show up for weekly session after the first meeting. Five participants remained active, although inconsistent with weekly attendance; 
reports on their weekly diet and exercise regimen were submitted to the program director via email or text. Participants experienced statistically significant reduction in weight with a $P$ value of $.043 \%$ using the non-parametric statistics. Figures of Mean in pre and post weight, are shown in appendix E, as followed $1.98 \%$ and $1.93 \%$. Confidence intervals for pre and post weight were $1.74 \%$ and $1.68 \%$ there were no statistical differences on the participants' waist circumference and BMI. The resulting $p$ value of both waist circumference and BMI respectively was $.068 \%$ and $.102 \%$. Perhaps if the program was completed longer than three months participants might have seen more changes in their weight. The program was conducted around the holidays (Thanksgiving, Christmas and New Years); perhaps timeframe of the program could be a factor in not seeing more significant changes in participants' waist and BMI. Additionally, the certified dietitian provided her education during the second month of the program as opposed of the beginning. Inconsistency in attendance was noted with some participants. However, the results were effective in showing no weight gain among all participants. Decrease in BMI was observed among three participants while two remained the same. There was no increase in BMI among any of the participants, which is a significant success for the program. The evidence in weight lost is clinically significant for the participants. Douketis, Macie, Thabane and Williamson, indicate that 5-10\% lost of baseline weight may improve, lipid, glucose and Hypertension. In obese people with cardiovascular risk factors, weight loss can provide some health benefits [16].

\section{Discussion}

"Leadership requires a level of self-knowledge and vulnerability that makes the growth experience visible to others" [17]. Commitment and effort are two of the characteristics of leaderships that a great leader should strive for. It is important to know that obstacles will arise unexpectedly, however with the foundation of hard work and persistence the leader will again prove himself or herself to his followers. The program coordinator was faced with overwhelming challenges during the course of getting site approval for the project. Adherence to effective communication either by phone or email was a concept adapted to keep stakeholders such as nurses, physicians, and therapists interested and engaged. They were informed about the project implementation process every time there were updates made to the approval step with ASU IRB in order to keep them aware of an anticipated date to start the program.

Participants were provided with information regarding the unexpected outcomes that could happen in the quest of reducing their weight through diet and exercise. Starvation was specifically noted to participants as a very unhealthy approach for weight lost. Participants were instructed to perform the light exercise movement learned from the weekly session as tolerate without doing strenuous exercise that could potentially injure their body.

When people engage in an ongoing activity and are informed of their performance attainments, some set goals for themselves spontaneously. Therefore, while revisiting one's obstacles in the quest to make changes, reinforcement on self-motivation should be reiterated in order for one to reach their destination.

Bandura indicated that in social cognitive theory (SCT) human behavior is extensively motivated and regulated by the ongoing exercise of self-influence. Models of behavior change also indicate that social and environmental factors play an important part in shaping attitudes. Obesity usually affects one's self-esteem and self-image, given the reason of why the choice of SCT was made for the educational program.
Figure of the SCT are displayed in appendix C. Due to the side effects of the medication, such as fatigue and drowsiness, the culture of the focus population is unintentionally inconsistent. As a result, some of the participants were not compliant with their weekly food journals as well as the hourly weekly meetings. Some of the reasons given to the program director for the number absences were forgetfulness and lack of interest.

Nurses are known to be in the forefront of the healthcare system, they are available 7 days a week, three hundred and sixty five days a year in the health care setting. Utilizing their expertise to promote and recruit participants was important in the initial stage of the project. Many patients became interested about the educational program simply from information given by their nurses on the unit. Nurses also had the opportunity to exhibit the information learned from the program coordinator, which can enrich their knowledge on current health problems affecting the health care system. The reason is why the Iowa model of evidence-based practice is important to nurses is because it provides them opportunities to explore evidence-based research regarding healthcare problems and initiate changes to the specific problem. An innovative method of communication was adapted to keep participants involved interested in the program. Weekly texting are sent to each participant as well as availability for individual meeting requested for specific questions from the program coordinator is allowed. The uniqueness of the innovation used, depended not only in assessing the progress of the participants, but mostly the communication engagement of the participants. They have the freedom to verbalize their choice about weekly food journals and exercise regimen. The program coordinator gives feedback regarding their choice on diet and exercise regimen. This feedback usually is referred back to the SCT, which motivates them to adhere to a healthy lifestyle.

Applying the Iowa model of evidence-based to share the project reports to the stakeholders regarding participants' feedback is an important aspect adapted in order to promote growth of an EBP culture in the organization. This model is adapted to expand stakeholder's knowledge and encourage EBP changes within the organization. The hospital administrators are very optimistic about incorporating the program guidelines into their curriculum in the near future as one of their therapeutic group meetings. Upon completion of the project, final results will be discussed with stakeholders hoping to have a concrete date to conduct the group merely based on this EBP project implementation this coming summer.

The specific steps mentioned above, translate both the model and logic adopted to explore health intervention through an educational program in the fight to decrease weight gain in the mental health setting. Obesity remains a serious threat in the health of many Americans. Patients taking antipsychotic medication are at even higher risk due to the metabolic effect. Changes are warranted from the providers to promote dietary education and moderate exercise regimen to patients in order to reduce the rate of the obesity epidemic.

Patients taking antipsychotic medication need to be placed on a standard healthy diet in order to prevent any unwanted weight gain. To accomplish this goal, patients must be aware of the lifestyle modification needed to prevent such risk. Moderate exercise and avoidance of sedentary lifestyle and poor nutrition are part of the treatment plan that providers should include when treating patients taking antipsychotic medication. These behavior changes in patients are imperative to be encouraged by providers. In addition, assessment 
of patients' knowledge level about their prescribed medications should be mandated in order to keep patients aware of the negative side effects such as weight gain.

\section{Conclusion}

The evidenced based practice project result shows positive impact on the healthcare system and behavioral health practice with a statistical significance of .043 decreased in participants' BMI. There are multiple medical diseases that are directly link to obesity. Diseases such as diabetes, cardiovascular system, and hypertension are often discussed among other diseases caused by obesity. These diseases will not be managed in-during a psychiatric visit. Psychiatrist providers will be focused on the management of the present mental illness, psychiatric medication education, and the importance of adherence to treatment. The psychiatrist will usually refer medical management to the primary care providers. It is emergent that obesity prevention is promoted in all healthcare institutions especially in the mental healthcare due to the direct impact of antipsychotic medications in decreasing patients' metabolism. Healthcare care spending can potentially be reduced, when extra tests, such as EKG, glucose monitoring are not needed. Data from Kaiser Family Foundation in 2009, indicates that $75 \%$ of the $\$ 2.2$ trillion spend by the United States on health care each year come from chronic diseases. It is our duty as providers and champions in the healthcare system to work as leaders to solve such complex problem. The integration of diet and exercise education is an important aspect of my practice when performing physical exam to patients. It is important to educate patients on weight management because chronic diseases such as, hypertension and diabetes is preventable by maintaining their weight at a normal level. Incorporating the result of this study can certainly affect patients in the behavioral health setting as well the primary care setting where I practice. Given the statistical significance of participants' BMI during the study, these findings can be used to convince policy makers that diet and exercise education can impact the healthcare system. One of the steps to disseminate the result of this project is to inform the stakeholders, especially the hospital administrators where this project was conducted, the positive results of this project. Secondly, giving speech on promoting diet and exercise education as a mandatory practice in the mental health setting, on a national, as well as international level conferences or seminars. To sustain the project, training will be provided to nurses, psychiatric providers, psychologists, therapists about safe diet and exercise regimen in order to assure that appropriate education is being delivered to patient by all disciplines involved in the patients' care. The guideline set for the study will be promoted, as an integral part of the education. It has proven to be effective in the reduction of BMI of the participants.

Failure on preventive medicine can directly impact the healthcare cost. For example, if diabetic patients do not adhere to their medication, it can cause 341,000 hospitalizations and 699,000 emergency department visits each year, resulting in annual spending of $\$ 5$ billion [18]. The numbers are real and the results are valid. We must do our part as providers to prevent such a deadly disease known to us all as obesity.

\section{References}

1. Ogden CL, Carroll MD, Kit BK, Flegal KM (2013) Prevalence of obesity in the United States: National Center for Health Statistics. NCHS data brief 82: (131).

2. Centers for Disease Control and Prevention (2013) Adult obesity facts. Retrieved from http://www.cdc.gov/obesity/data/adult.html

3. Park T, Usher K, Foster K (2011) Description of a healthy lifestyle intervention for people with serious mental illness taking second-generation antipsychotics. Int J Ment Health Nurs 20: 428-437. [Crossref]

4. Menifield CE, Dothy N, Fletcher A (2008) Obesity in America. The ABNF J: 83-88

5. McLaughlin M (2012) Knowledge compassion focused on you. Med Star Health: 1-49.

6. Wu RR, Zhao JP, Jin H, Shao P, Fang MS, et al. (2008) Lifestyle intervention and metformin for treatment of antipsychotic-induced weight gain. JAMA 299: 185-193. [Crossref]

7. Newman SC, Bland RC (1991) Mortality in a cohort of patients with schizophrenia: a record lineage study. Can J Psychiatry 36: 239-245. [Crossref]

8. Jay M, Kalet A, Ark T, McMacken M, Messito MJ (2009) Physicians' attitudes about obesity and their associations with competency and specialty: A cross-sectional study. BMC Health Serv Res 9. [Crossref]

9. Melamed Y, Steln-Reisner O (2008) Multi-modal weight control intervention for people with persistent mental disorders. Psych Rehab J 31: 194-200. [Crossref]

10. Prochaska JO, DiClemente CC (1984) The transtheoretical approach: Crossing traditional boundaries of therapy. Dow Jones Irwin, Homewood, IL.

11. Prochaska JO, DiClemente CC (1986) Towards a comprehensive model of change. Treating addictive behaviors: Processes of Change 3-27.

12. Moulding, NT, Silagy CA, Weller DP (2013) A framework for effective management of change in clinical practice: Dissemination and implementation of clinical practice guidelines. Quality in Health Care

13. White J, Gray R, Jones M (2009) The development of the serious mental illness physical health improvement profile. J Psychiatr Ment Health Nurs 16: 493-498. [Crossref]

14. Institute of medicine (2004) Data sources on health literacy: A prescription to end confusion available from the national academies press: Washington, DC: National Academies Press.

15. Hargittai E (2002) Second-level digital divide: Differences in people's online skills First Monday 5(4).

16. Douketis JD, Macie C, Thabane L, Williamson DF (2005) Systematic review of longterm weight loss studies in obese adults: clinical significance and applicability to clinical practice. Int J Obes (Lond) 29: 1153-1167. [Crossref]

17. Porter-O'Grady T, Malloch K (2011) Quantum leadership: Advancing information, transforming health care ( 3 rd ed). Sudbury, MA: Jones \& Bartlett.

18. Jah AK, Aubert RE, Yao J, Teagarden JR, Epstein RS (2012) "Greater adherence to diabetes drugs is linked to less hospital use and could save nearly $\$ 5$ billion annually." Health Affairs 31: 1836-1846. [Crossref]

Copyright: (C2016 Aurelus E. This is an open-access article distributed under the terms of the Creative Commons Attribution License, which permits unrestricted use, distribution, and reproduction in any medium, provided the original author and source are credited. 